\title{
Anti-infectious and Anti-tumor Activities of $\boldsymbol{\beta}$-glucans
}

\author{
VACLAV VETVICKA and JANA VETVICKOVA \\ Department of Pathology, University of Louisville, Louisville, KY, U.S.A.
}

\begin{abstract}
Background/Aim: The aim of this study was to directly compare the anti-infectious and anti-cancer effects of five commercially available glucans. Materials and Methods: We used five different glucans isolated from algae, yeast, bacteria, oat, and mushroom. We compared their effects on the stimulation of phagocytosis of blood cells, on the secretion of $I L-2$, and on the inhibition of melanoma and breast and lung cancers. In addition, we evaluated the effects of glucan supplementation on two experimental models of infection. Results: Most of the tested glucans stimulated phagocytosis and IL-2 secretion, reduced cancer growth, and ameliorated some effects of experimental infections. Conclusion: Glucans can produce significant pleiotropic effects, but the activity varies among individual samples.
\end{abstract}

$\beta$-Glucans (hereafter called "glucans") are well-established natural immunomodulators with documented history of research reaching 60 years back, when a crude yeast extract was discovered to stimulate macrophages by complement activation (1). Glucans were found to be active throughout the animal kingdom and so far, no species has been found to be glucan resistant. Immunostimulating effects of glucans have been demonstrated in shrimp, fish (2), chicken (3), mice, rats, rabbits, goats, sheep, pigs (4), apes (5), and humans [for a review, see Vetvicka 2013 (6)]. Well-established biological effects of glucans include stimulation of immune reactions (7), control of diabetes (8), reduction of chronic fatigue syndrome (9), lowering of cholesterol levels $(10,11)$, and inhibition of cancer development (12).

With numerous natural sources of glucans, including yeast, mushroom, bacteria, seaweed, and grain, it is not surprising that hundreds of structurally and functionally different glucans exist. However, despite the significant

This article is freely accessible online.

Correspondence to: Vaclav Vetvicka, University of Louisville, Department of Pathology, 511 S. Floyd, Louisville, KY 40202, U.S.A. Tel: +1 5028521612, e-mail: Vaclav.vetvicka@louisville.edu

Key Words: Glucan, cancer, phagocytosis, IL-2, infection. efforts of many laboratories, a direct link between structure and function remains elusive (13-15).

Currently, more than 30,000 studies on the biological actions of glucans can be found in the peer-reviewed literature. However, there are still problems with results not being fully comparable, as dozens and most probably hundreds of glucans exist, most commercially available. With significant differences in source, purity, physicochemical characteristics, and solubility, it is often difficult to reach a conclusion regarding the general effects of glucans. The only viable solution is to perform direct comparative evaluations; hence, we endeavored to directly compare the anti-infectious and anti-cancer effects of five commercially available glucans.

\section{Materials and Methods}

Animals. Female, 8-week-old BALB/c mice were purchased from the Jackson Laboratory (Bar Harbor, ME, USA). All animal work was performed according to the University of Louisville IACUC protocol. Animals were sacrificed by $\mathrm{CO}_{2}$ asphyxiation followed by cervical dislocation.

Materials. All glucans were either donated or purchased from the manufacturers or distributors as shown in Table I. Concanavalin A (Con A), RPMI 1640 medium, fetal calf serum (FCS), polymyxin B, Escherichia coli, and Wright stain were purchased from Sigma (St. Louis, MO, USA). Levine EMB (eosin methylene blue) agar and Tryptic Soy Broth were purchased from Fisher Scientific (Waltham, MA, USA). Pseudomonas aeruginosa (ATCC 27853) was purchased from ATT (Manassas, VA, USA).

Cell lines. Murine melanoma B16 cell line (ATCC, Manassas, VA), Lewis lung carcinoma cells (obtained from Dr. G. Ross, University of Louisville, Louisville, KY, USA), and BALB/c mouse-derived mammary tumor cell line Ptas64 (generously provided by Dr. Wei-Zen Wei of the Michigan Cancer Foundation, Detroit, MI, USA) were maintained in culture at $37^{\circ} \mathrm{C}$ in a humidified atmosphere supplemented with $5 \% \mathrm{CO}_{2}$ in RPMI 1640 medium supplemented with $10 \%$ FCS.

Phagocytosis. Phagocytosis of synthetic polymeric microspheres has been described previously (16). Briefly, $0.1 \mathrm{ml}$ of peripheral blood from mice injected with various doses of glucan or PBS were incubated in vitro with $0.05 \mathrm{ml}$ of 2-hydroxyethyl methacrylate particles $\left(5 \times 10^{8} / \mathrm{ml}\right)$. The tubes were incubated at $37^{\circ} \mathrm{C}$ for $60 \mathrm{~min}$, with intermittent shaking. Smears were stained with Wright stain. The cells with three or more particles were considered positive. Mice were injected with either glucan or PBS (negative control). 
Table I. List of glucans used in this study.

\begin{tabular}{lccc}
\hline Glucan name & Source & Solubility & Manufacturer \\
\hline Glucan \#300 & Yeast & Insoluble & Transfer Point, Little Mountain, SC, USA \\
Super Pure Beta 1,3 Glucan Algae Extract & Algae & Insoluble & The Synergy Company, Moab, UT, USA \\
Glucan Elite & Bacterial & Semisoluble & Pro Formulations MD, Orange, CA, USA \\
Beta Glucan & Oat & Semisoluble & Bioimersion, Bellevue, WA, USA \\
Maitake Gold & Mushroom & Soluble & NutraGenesis, Brattleboro, VT, USA \\
\hline
\end{tabular}

All experiments were performed in triplicate. At least 300 cells were examined in each experiment.

$I L-2$ secretion. Purified spleen cells $\left(2 \times 10^{6} / \mathrm{ml}\right.$ in RPMI 1640 medium with $5 \%$ FCS) obtained from mice injected with $100 \mu \mathrm{g}$ glucan or PBS were added into wells of a 24 -well tissue culture plate. Cells were incubated for $48 \mathrm{~h}$ in a humidified incubator $\left(37^{\circ} \mathrm{C}, 5 \% \mathrm{CO}_{2} / 95 \%\right.$ air). Addition of $1 \mu \mathrm{g}$ of Concanavalin $\mathrm{A}$ (Sigma) was used as a positive control. At the endpoint of incubation, supernatants were collected, filtered through $0.45 \mu \mathrm{m}$ filters, and tested for the presence of IL-2 using a Quantikine mouse IL-2 kit (R\&D Systems, Minneapolis, MN, USA).

Tumor inhibition in vivo. Mouse mammary, lung, and melanoma cancer models have been described previously in full detail (12).

Urinary tract infection. Urinary tract infection was induced via direct inoculation of $10 \mu \mathrm{l}$ of $E$. coli into the bladder using a syringe equipped with soft polyethylene tubing as previously described (17, 18). Tested glucans were used orally at $100 \mu \mathrm{g}$ /mouse doses for 14 days. Mice were sacrificed at various intervals $(6 \mathrm{~h}, 24 \mathrm{~h}$, and $72 \mathrm{~h})$ after infection and bladder and right kidney were aseptically collected from each. After weighing, organs were homogenized in sterile PBS. Obtained material was centrifuged to discard the tissue fragments. Appropriate dilutions were plated on Petri dishes with Levine EMB agar (Fisher Scientific (Waltham, MA, USA) and incubated at $37^{\circ} \mathrm{C}$. Colony-forming units (CFU) were evaluated $24 \mathrm{~h}$ later.

P. aeruginosa. $P$. aeruginosa was incubated at $37^{\circ} \mathrm{C}$ overnight on $5 \%$ sheep blood agar plates. Colonies were grown overnight in Tryptic Soy Broth. Bacteria were centrifuged at 4,000 $\times g$ washed with PBS, and suspended to a density of $1 \times 10^{8} \mathrm{CFU} / \mathrm{ml}$. The density for both instillation and bronchoalveolar lavage fluid (BALF) collection were determined by serial culture dilutions. Pneumonia was induced by intranasal instillation of $10^{6} \mathrm{CFU}$ of $P$. aeruginosa. Three days later, BALF was collected and bacterial counts and neutrophil counts were performed (19).

Statistical analysis. Statistical significance was evaluated by paired $t$-test and by unpaired $t$-test using a GraphPad Prism 502 software (GraphPad Software, San Diego, CA, USA).

\section{Results}

Commercial samples, particularly when used from broken capsules, might contain small amounts of fillers such as gelatin or microcrystalline cellulose. These fillers are biologically inert and the risk of influencing tested biological reactions is only theoretical. In addition, they might slightly lower the dose of the tested material, as the amount of the tested material in the capsule is slightly lower than the amount on the bottle. However, our previous experiments showed that direct comparison of one particular glucan obtained either from a commercial capsule or directly from pure glucan powder yielded identical results (12). Another possible way to achieve defective results is possible contamination with lipopolysaccharide. We prepared lipopolysaccharide-free samples and compared their effects on phagocytosis but found no differences from untreated samples (data not shown). For our experiments, we have chosen five commercially available glucans (Table I). Glucan \#1 is one of the most studied glucans (20), glucan \#2 is an insoluble glucan isolated from algae, glucan \#3 is Glucan Elite, an Agrobacterium-derived glucan (21), glucan \#4 is a semisoluble oat-derived glucan, and glucan \#5 is Maitake Gold isolated from mushrooms (22).

The glucans were originally used as nonspecific immunomodulators of cellular immunity. We decided to test their ability to stimulate phagocytosis of peripheral blood neutrophils. The glucans \#1,\#2, \#3, and \#5 had significant effects on phagocytic activity, with glucan \#1 showing the highest effects (Figure 1).

Next, we focused on the evaluation of the effects of glucans on the secretion of IL-2 by splenocytes. The basal levels of IL-2 without any stimulation are almost zero. All glucans showed significant stimulation of IL-2 secretion, which was comparable to the effects of Con A (Figure 2).

After establishing the basic effects on some immune reactions, we focused on their effects on cancer development. We used two different experimental models of tumor growth. Using a breast cancer model, we evaluated the changes in tumor weight. Our data (Figure 3) showed that the tested glucans again differed in their activity towards breast cancer inhibition and only glucans \#1, \#2, and \#5 significantly suppressed the growth of breast cancer. Next, we used a well-described Lewis lung carcinoma assay. Our data (Figure 4) confirmed the breast cancer experiments, with additional suppression being found in using glucan \#3. Again, glucan \#1 showed the highest effects. Similar results were found in case of melanoma inhibition (Figure 5). 


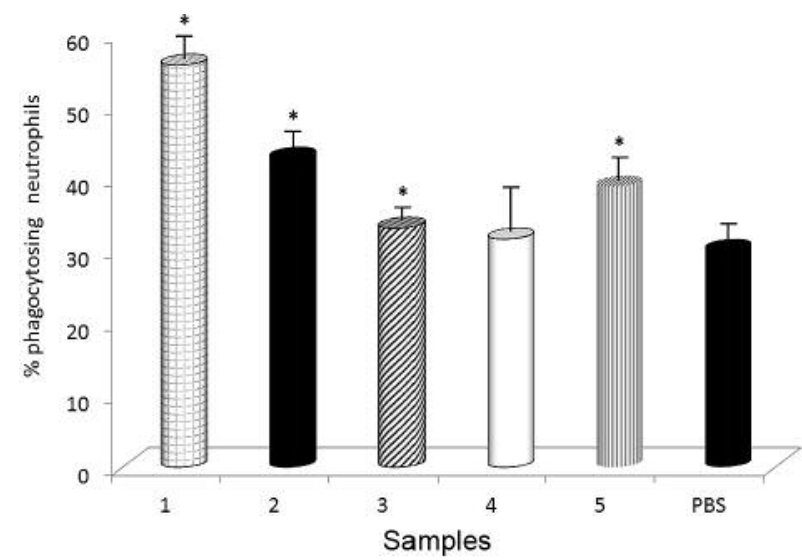

Figure 1. Effects of glucan treatment on the phagocytosis of microparticles by peripheral blood neutrophils. The experiments were repeated three times, results are given as mean $\pm S D$. *Significant at $p<0.05$.

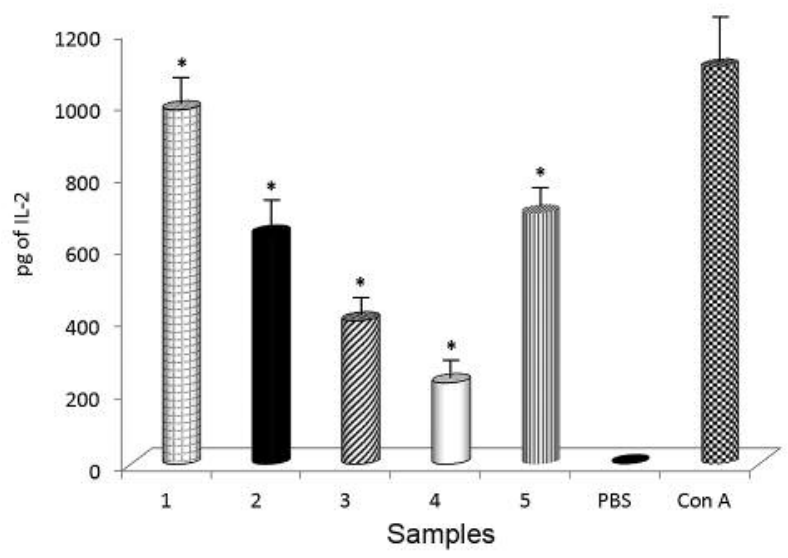

Figure 2. Effects of the tested glucans on the secretion of IL-2 by spleen cells. As the control (PBS) production of IL-2 is not detectable, all columns represent significant differences between control $(P B S)$ and samples $(p<0.05)$. All experiments were performed in triplicates.

Next, we focused on the antibacterial effects. Pneumonia was induced by intranasal application of $10^{6} \mathrm{CFU}$ of P. aeruginosa. Three days later, BALF was collected and bacterial counts and neutrophil counts were performed. In the pneumonia conditions, the BALF bacterial counts were significantly lower in animals treated with glucans \#1, \#2, \#3, and \#5, with glucans \#1 and \#3 being the most active (Figure 6). When we measured the changes in the numbers of neutrophils in BALF, all glucans suppressed their numbers (Figure 7).

Using a model of mouse urinary tract infection, we observed the appearance of $E$. coli as CFU in bladder after 14 days of oral treatment. Figure 8 shows that four of the tested glucans significantly decreased the amount of E. coli

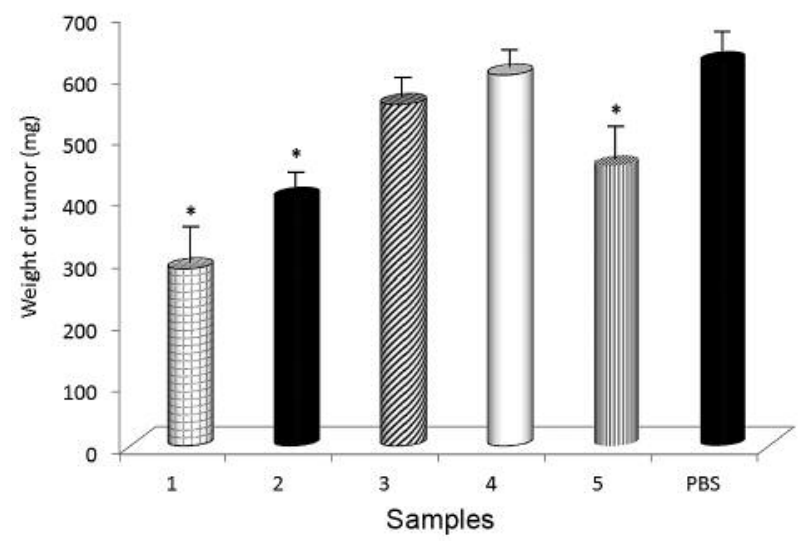

Figure 3. Effects of glucan supplementation on the weight of primary breast cancer tumors. Results represent mean from three experiments $\pm S D$. *Represents significant differences from control, $p<0.05$.

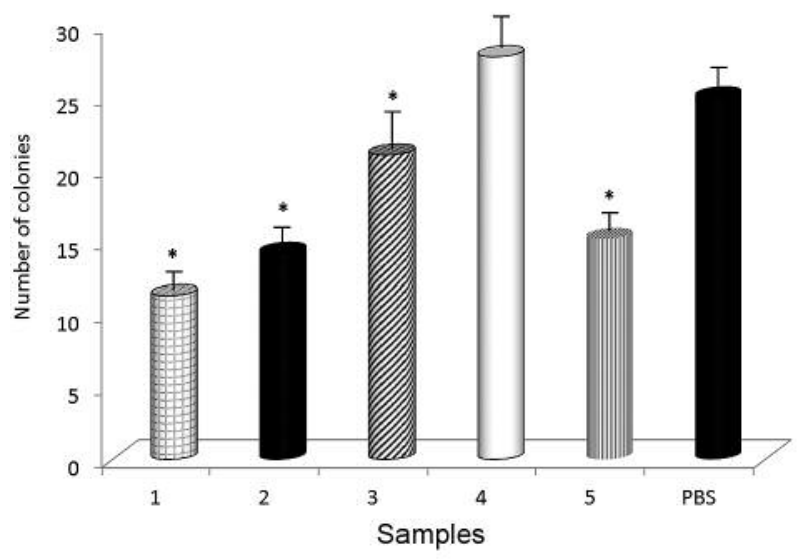

Figure 4. Effects of glucan supplementation on the number of lung colonies. Results represent mean from three experiments $\pm S D$. $*$ Represents significant differences from control, $p<0.05$.

in the bladder of tested animals in both tested intervals $(24 \mathrm{~h}$ and $72 \mathrm{~h}$ ). Furthermore, we found that all glucans significantly reduced the number of bacteria in the kidney samples after 3 days post infection. The results taken 7 days after infection were similar but, in this case, glucan \#4 showed only a small decrease of CFU (Figure 9).

\section{Discussion}

The immunomodifying effects of glucans are well established. From the pioneering work of Nicolas DiLuzio, who demonstrated significant stimulation of macrophages, improved defense reactions, and tumor resistance (23), 


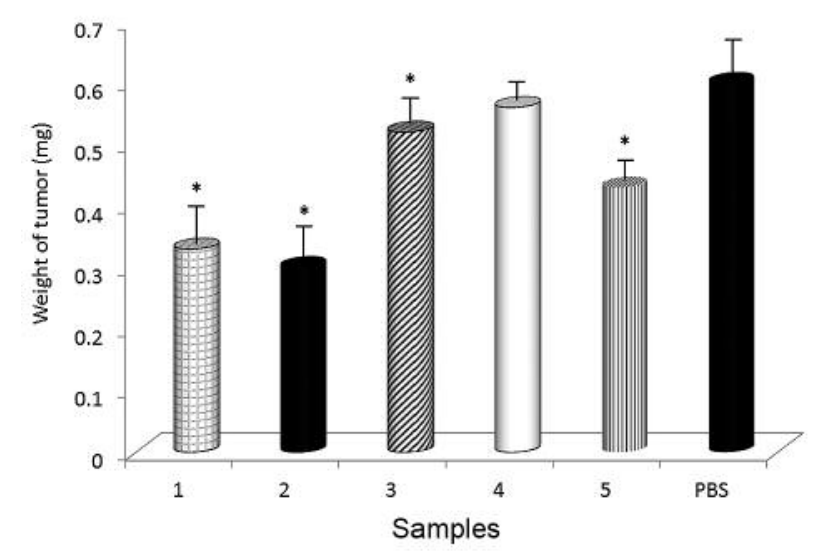

Figure 5. Effects of glucan supplementation on the weight of melanoma tumors. Results represent mean from three experiments $\pm S D$. *Represents significant differences from control, $p<0.05$.

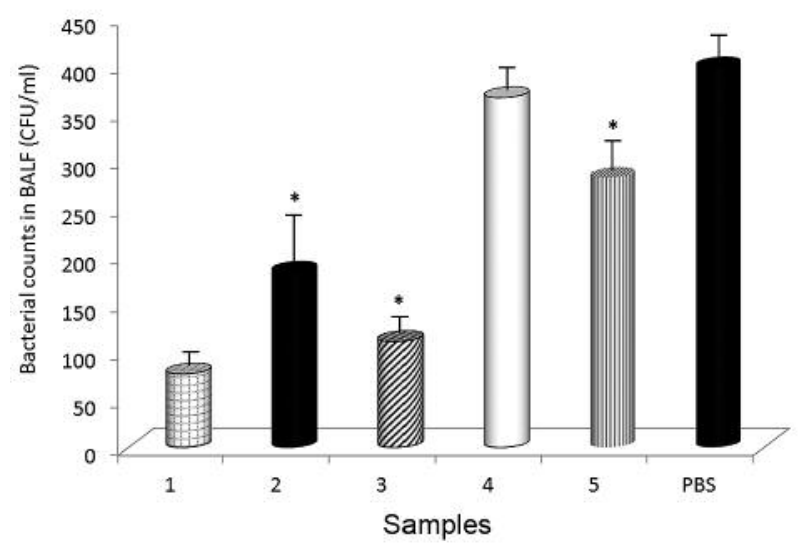

Figure 6. Effects of glucan supplementation on bacteria counts in BALF. Results represent mean from three experiments $\pm S D$. *Represents significant differences from control, $p<0.05$.

decades of intensive research performed all around the world have confirmed that glucans represent the most active natural immunomodulator. Glucan effects are manifested via binding to several specific receptors, such as CR3 and dectin-1, and subsequent glucan-related signaling pathways include the NFkB-receptor tyrosine kinase axis (24). Their effects include not only improvements of immune reactions against cancer and infection $(7,25)$, but also protection against mitochondrial toxicity (26) or improvements in diabetic retinopathy (27). Recently, glucans have been evaluated for their use in vaccine preparation, either as a part of the targeting delivery system or as a nanoparticle-based delivery platform (28).

Despite the fact that glucans are the most studied natural immunomodulators and have been an approved as drugs in Japan for decades (29), they are not without problems. Several

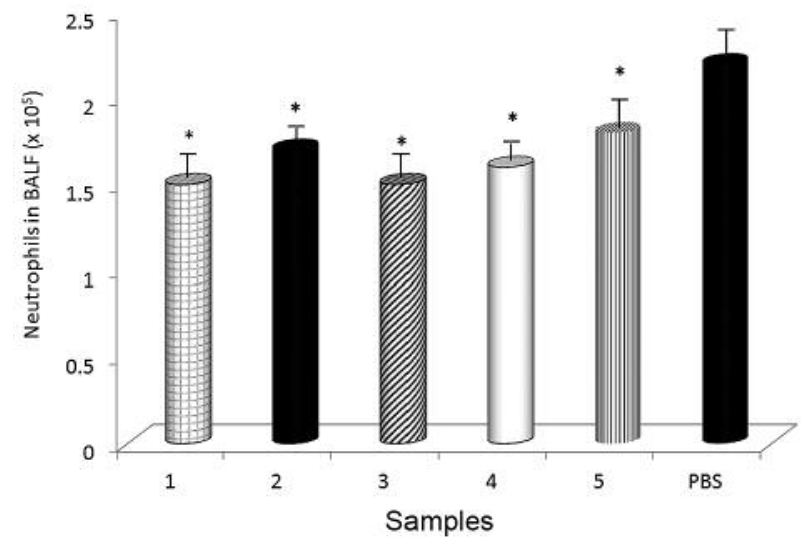

Figure 7. Effects of glucan supplementation on neutrophil counts in $B A L F$. Results represent mean from three experiments $\pm S D$. * Represents significant differences from control, $p<0.05$.

studies have examined the effects of glucans, however, direct comparison of their effects is difficult. The use of synthetic glucan-based oligosaccharides was expected to overcome these problems, but their use is limited due to the difficult and expensive preparation (30). To better understand the biological effects of glucans and to further increase the chance that glucans are used in Western medicine, it is important to directly compare glucans using identical methods. Our previous studies compared over 60 individual glucans $(12,31$, 32 ), but the ever increasing multiplication of new commercial glucans led us to compare some of the new glucans with glucan \#300, which has been previously shown to be superior.

Glucans were originally considered to be nonspecific stimulators of cellular immunity; therefore, phagocytosis was and still is the test of choice, because the universal dogma stating that glucans not stimulating phagocytosis will not affect immunity is still valid. The effects of glucans on phagocytosis are mediated via interaction with glucan receptors (6). Here, we used synthetic microbeads based on 2-hydroxyethyl methacrylate with demonstrated low spontaneous adhesion to the cell surface and, therefore, low false positivity (16). Our results demonstrated that glucans really stimulate phagocytosis of professional phagocytes, but that there are significant differences in the activities among the tested glucans.

In order to show that glucans affect additional facets of the immune system, we evaluated the potential stimulation of IL2 secretion. The relationship between glucans and cytokines is complicated. On one hand, high systemic stimulation of secretion of many cytokines is not desirable; on the other hand, there is only one glucan described in literature as having no effects on cytokine secretion (33). We confirmed that all glucans significantly increased IL-2 secretion by spleen cells. However, it is important to note that without any stimulation, splenocytes do not secrete IL-2. 


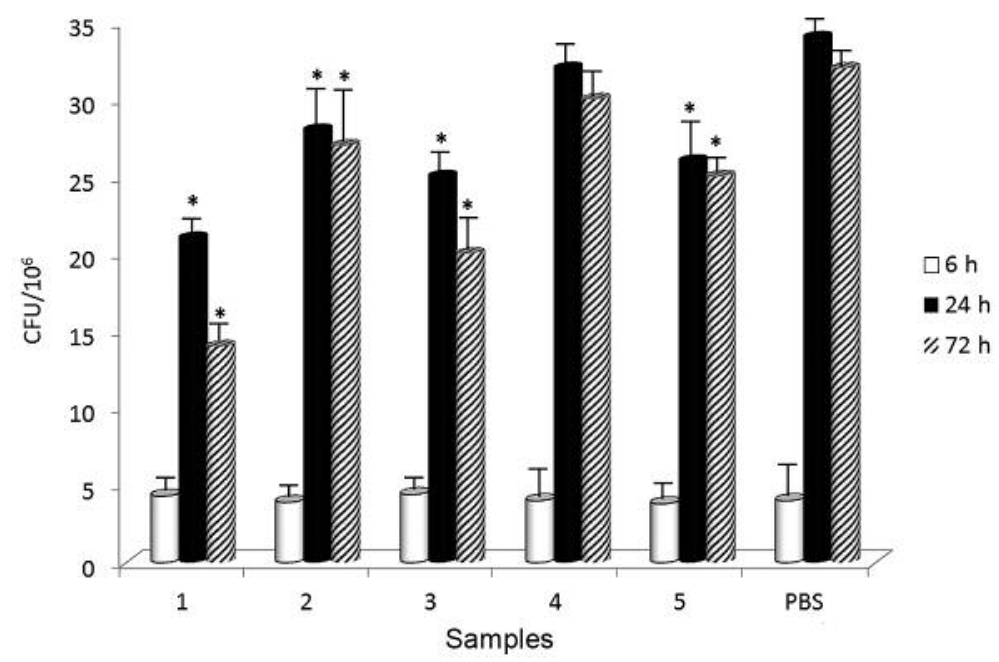

Figure 8. Effects of glucan supplementation on CFU in the bladder at different time intervals. Results represent mean from three experiments $\pm S D$. *Represents significant differences from control, $p<0.05$.

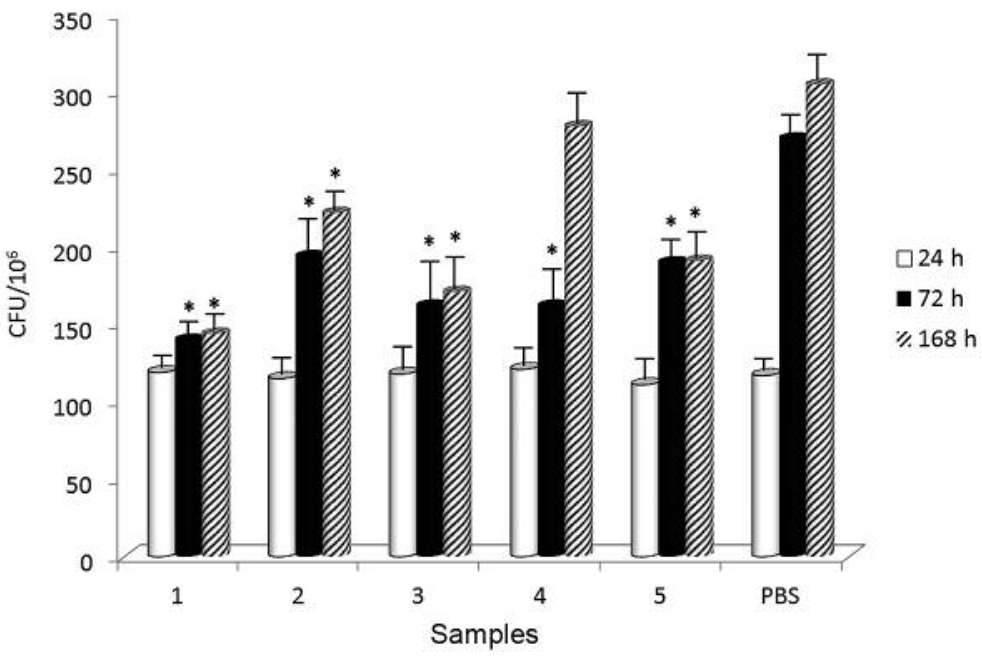

Figure 9. Effects of glucan supplementation on CFU in kidney at different time intervals. Results represent mean from three experiments $\pm S D$. *Represents significant differences from control, $p<0.05$.

Glucans are well-established natural immunomodulators with significant anti-cancer properties (34). Glucans can either directly inhibit cancer cell growth or stimulate cells such as NK cells or neutrophils to kill cancer cells upon binding to glucan receptors. In addition, some novel roles of glucan in cancer treatment have recently been described, ranging from glucans being a delivery platform for anticancer drugs (35) to regulating trained immunity, where innate cells take on memory phenotypes. To better compare the effects of glucan supplementation on cancer development, we used three different models of breast cancer, lung cancer, and melanoma. Our data showed that most of glucans inhibit cancer development by reducing the primary tumor weight and the number of lung cancer colonies. Glucan \#4, however, showed only small, nonsignificant inhibition. Based on our previous data, we hypothesized that these effects are mediated by a combination of stimulation of effector cells, such as NK cells, and general stimulation of immune system (20).

Similar to cancer, glucans have also been established as anti-infectious agents $(36,37)$. In our comparative study, we used two different models of infection. First, we focused on urinary tract infection, as it represents a common clinical problem. Repeated infections are, at least partly, caused by 
depressed immune system. This infection includes both infection of the kidney and infection of the bladder and is most commonly caused by $E$. coli.

Using a model of mouse urinary tract infection, we counted the CFU of E. coli in the bladder after 14 days of oral treatment and found that four of the tested glucans significantly decreased the amount of $E$. coli at both intervals ( $24 \mathrm{~h}$ and $72 \mathrm{~h}$ ). When we measured the number of bacteria in the kidney, we found that all glucans significantly reduced their numbers after 3 days post infection. All glucans were active, but significant differences among glucans mirrored those found in previous tests.

The second experimental infection model was a mouse model of pneumonia. We chose this model because bacterialcaused pneumonia is a leading cause of acute respiratory distress syndrome and significantly increases the mortality of patients in intensive care units (38). Food supplementation with glucan resulted in significant depression of high neutrophil counts caused by infection. In addition, four out of the five tested glucans reduced bacterial counts in BALF. This model of $P$. aeruginosa infection is highly relevant to clinical situation (39) and our results suggest the potential use of glucans as a supportive treatment in pneumonia patients.

\section{Conclusion}

In conclusion, this study is a follow-up of our previous comparative studies testing over 60 different glucans. From the current and previous studies, we can clearly confirm that glucans have significant immuno-stimulating, anti-infectious, and anti-cancer effects. Furthermore, we clearly showed that there are huge differences in activities among the individual glucans and that the source of glucan is not the most important factor responsible for glucan effects. Current literature on glucan often yields contradictory results. Based on our results, we believe that the most probable explanation are the differences in biological activities among individual glucans. However, glucan \#300 remains the most active glucan.

\section{Conflicts of Interest}

The Authors declare that there are no conflicts of interests related to this study.

\section{Authors' Contributions}

VV designed the project, supervised the experiments, analyzed data and wrote the manuscript. JV performed the experiments, prepared the Figures and co-wrote the manuscript.

\section{Acknowledgements}

The Authors are grateful to Ms. Tracey Bender for her editorial help.

\section{References}

1 Benacerraf B and Sebestyen MM: Effect of bacterial endotoxins on the reticuloendothelial system. Fed Proc 16(3): 860-867, 1957. PMID: 13480376.

2 Vetvicka V, Vannucci L and Sima P: The effects of beta-glucan on fish immunity. N Am J Med Sci 5(10): 580-588, 2013. PMID: 24350069. DOI: 10.4103/1947-2714.120792

3 Shao Y, Wang Z, Tian X, Guo Y and Zhang H: Yeast beta-d-glucans induced antimicrobial peptide expressions against salmonella infection in broiler chickens. Int J Biol Macromol 85: 573-584, 2016. PMID: 26794312. DOI: 10.1016/j.ijbiomac.2016.01.031

4 de Vries S, Gerrits WJ, Kabel MA, Vasanthan T and Zijlstra RT: Beta-glucans and resistant starch alter the fermentation of recalcitrant fibers in growing pigs. PLoS One 11(12): e0167624, 2016. PMID: 27911928. DOI: 10.1371/journal.pone.017624

5 Reynolds JA, Kastello MD, Harrington DG, Crabbs CL, Peters CJ, Jemski JV, Scott GH and Di Luzio NR: Glucan-induced enhancement of host resistance to selected infectious diseases. Infect Immun 30(1): 51-57, 1980. PMID: 7439978.

6 Větvička Va: b-glucans as natural biological response modifiers. Nova Science Publishers, Inc. New York, 2013.

7 Novak $\mathrm{M}$ and Vetvicka V: Beta-glucans, history, and the present: Immunomodulatory aspects and mechanisms of action. J Immunotoxicol 5(1): 47-57, 2008. PMID: 8382858. DOI: 10.1080/15476910802019045

8 Wursch P and Pi-Sunyer FX: The role of viscous soluble fiber in the metabolic control of diabetes. A review with special emphasis on cereals rich in beta-glucan. Diabetes Care 20(11): 1774-1780, 1997. PMID: 9353622. DOI: 10.2337/diacare.20.11.1774

9 Richter J, Kral V, Stiborova I, Rajnohova D and Vetvicka V: Anti-inflammatory effects of $\beta$-glucan in cancer related fatigue. J Nutr Health Sci 2(3): 304, 2015. DOI: 10.15744/23939060.2.304

10 Henrion M, Francey C, Le KA and Lamothe L: Cereal b-glucans: The impact of processing and how it affects physiological responses. Nutrients 11(8), 2019. PMID: 31357461.

11 Sima P, Vannucci L and Vetvicka V: b-glucans and cholesterol (review). Int J Mol Med 41(4): 1799-1808, 2018. PMID: 29393350. DOI: 10.3892/ijmm.2018.3411

12 Vetvicka V and Vetvickova J: Glucans and cancer: Comparison of commercially available b-glucans - part IV. Anticancer Res 38(3): 1327-1333, 2018. PMID: 29491056. DOI: 10.21873/anticanres. 12355

13 Bae IY, Kim HW, Yoo HJ, Kim ES, Lee S, Park DY and Lee HG: Correlation of branching structure of mushroom $\beta$-glucan with its physiological activities. Food Res Int 51(1): 195-200, 2013. DOI: $10.1016 /$ j.foodres.2012.12.008

14 Novak M and Vetvicka V: Development of views on $\beta$-glucan composition and structure. In: Biology and Chemistry of Beta Glucan, Vetvicka V, Novak M (eds.). Bentham Sci Publ, pp. 19, 2011 .

15 Ohno N, Miura NN, Chiba N, Adachi Y and Yadomae T: Comparison of the immunopharmacological activities of triple and single-helical schizophyllan in mice. Biol Pharm Bull 18(9): 1242-1247, 1995. PMID: 8845814. DOI: 10.1248/bpb.18.1242

16 Vetvicka V, Fornusek L, Kopecek J, Kaminkova J, Kasparek L and Vranova M: Phagocytosis of human blood leukocytes: A simple micromethod. Immunol Lett 5(2): 97-100, 1982. PMID: 7152553. DOI: 10.1016/0165-2478(82)90040-2 
17 Hopkins WJ, Hall JA, Conway BP and Uehling DT: Induction of urinary tract infection by intraurethral inoculation with escherichia coli: Refining the murine model. J Infect Dis 171(2): 462-465, 1995. PMID: 7844391. DOI: 10.1093/infdis/171.2.462

18 Rosen DA, Hung CS, Kline KA and Hultgren SJ: Streptozocininduced diabetic mouse model of urinary tract infection. Infect Immun 76(9): 4290-4298, 2008. PMID: 18644886. DOI: 10.1128/IAI.00255-08

19 Zheng J, Huang Y, Islam D, Wen XY, Wu S, Streutker C, Luo A, Li M, Khang J, Han B, Zhong N, Li Y, Yu K and Zhang H: Dual effects of human neutrophil peptides in a mouse model of pneumonia and ventilator-induced lung injury. Respir Res 19(1): 190, 2018. PMID: 30268129. DOI: 10.1186/s12931-018-0869-x

20 Vetvicka V and Vetvickova J: Glucan supplementation has strong anti-melanoma effects: Role of NK cells. Anticancer Res 35(10): 5287-5292, 2015. PMID: 26408688.

21 Xu X, Ding Y, Yang Y, Gao Y, Sun Q, Liu J, Yang X, Wang J and Zhang $\mathrm{J}$ : Beta-glucan salecan improves exercise performance and displays anti-fatigue effects through regulating energy metabolism and oxidative stress in mice. Nutrients 10(7), 2018. PMID: 29970808. DOI: 10.3390/nu10070858

22 Roldan-Deamicis A, Alonso E, Brie B, Braico DA and Balogh GA: Maitake pro4x has anti-cancer activity and prevents oncogenesis in balbc mice. Cancer Med 5(9): 2427-2441, 2016 PMID: 27401257.

23 Di Luzio NR and Riggi SJ: The effects of laminarin, sulfated glucan and oligosaccharides of glucan on reticuloendothelial activity. J Reticuloendothel Soc 8(5): 465-473, 1970. PMID: 5471976.

24 Roy S, Dickerson R, Khanna S, Collard E, Gnyawali U, Gordillo GM and Sen CK: Particulate beta-glucan induces tnf-alpha production in wound macrophages via a redox-sensitive nfkappabeta-dependent pathway. Wound Repair Regen 19(3): 411419, 2011. PMID: 21518092. DOI: 10.1111/j.1524-475X.2011. 00688.x

25 Novak $M$ and Vetvicka V: Glucans as biological response modifiers. Endocr Metab Immune Disord Drug Targets 9(1): 6775, 2009. PMID: 19275682 . DOI: $10.2174 / 187153009787582423$

26 Shaki F and Pourahmad J: Mitochondrial toxicity of depleted uranium: Protection by beta-glucan. Iran J Pharm Res 12(1): 131-140, 2013. PMID: 24250581.

27 Richter J, Zavorkova M, Stiborova I, Kral V and Vetvicka V: Diabetic retinopathy: Changes in levels of orosomucoids in patients supplemented with beta glucan and vitamin D. Am J Immunol 14: 26-33, 2019. DOI: 10.3844/ajisp.2018.26.33

28 Vetvicka V, Vannucci L and Sima P: b-glucan as a new tool in vaccine development. Scand J Immunol: e12833, 2019. DOI: $10.1111 /$ sji.12833

29 Ina K, Kataoka $\mathrm{T}$ and Ando T: The use of lentinan for treating gastric cancer. Anticancer Agents Med Chem 13(5): 681-688, 2013. PMID: 23092289. DOI: $10.2174 / 1871520611313050002$
30 Sylla B, Guegan JP, Wieruszeski JM, Nugier-Chauvin C, Legentil L, Daniellou R and Ferrieres V: Probing b-(1->3)-dglucans interactions with recombinant human receptors using high-resolution nmr studies. Carbohydr Res 346(12): 1490-1494, 2011. DOI: 10.1016/j.carres.2011.03.038

31 Vetvicka V and Vetvickova J: Physiological effects of different types of beta-glucan. Biomed Pap Med Fac Univ Palacky Olomouc Czech Repub 151(2): 225-231, 2007. PMID: 18345255. DOI: $10.5507 / \mathrm{bp} .2007 .038$

32 Vetvicka V and Vetvickova J: Comparison of immunological effects of commercially available b-glucans. Appl Sci Rep 1: 17, 2014. DOI: 10.7243/2054-9903-1-2

33 Patchen ML, Liang J, Vaudrain T, Martin T, Melican D, Zhong S, Stewart M and Quesenberry PJ: Mobilization of peripheral blood progenitor cells by betafectin pgg-glucan alone and in combination with granulocyte colony-stimulating factor. Stem Cells 16(3): 208217, 1998. PMID: 9617896. DOI: 10.1002/stem.160208

34 Geller A, Shrestha R and Yan J: Yeast-derived b-glucan in cancer: Novel uses of a traditional therapeutic. Int J Mol Sci 20(15), 2019. PMID: 31344853. DOI: 10.3390/ijms20153618

35 Elder MJ, Webster SJ, Chee R, Williams DL, Hill Gaston JS and Goodall JC: Beta-glucan size controls dectin-1-mediated immune responses in human dendritic cells by regulating IL1beta production. Front Immunol 8: 791, 2017. PMID: 28736555. DOI: $10.3389 /$ fimmu.2017.00791

36 Dharsono T, Rudnicka K, Wilhelm M and Schoen C: Effects of yeast $(1,3)-(1,6)$-beta-glucan on severity of upper respiratory tract infections: A double-blind, randomized, placebo-controlled study in healthy subjects. J Am Coll Nutr 38(1): 40-50, 2019. PMID: 30198828. DOI: 10.1080/07315724.2018.1478339

37 Mah E, Kaden VN, Kelley KM and Liska DJ: Soluble and insoluble yeast beta-glucan differentially affect upper respiratory tract infection in marathon runners: A double-blind, randomized placebo-controlled trial. J Med Food 23(4): 416-419, 2019. PMID: 31573387. DOI: 10.1089/jmf.2019.0076

38 Vincent JL, Rello J, Marshall J, Silva E, Anzueto A, Martin CD, Moreno R, Lipman J, Gomersall C, Sakr Y, Reinhart K and Investigators EIGo: International study of the prevalence and outcomes of infection in intensive care units. JAMA 302(21): 23232329, 2009. PMID: 19952319. DOI: 10.1001/jama.2009.1754

39 Sawa T, Corry DB, Gropper MA, Ohara M, Kurahashi K and Wiener-Kronish JP: Il-10 improves lung injury and survival in Pseudomonas aeruginosa pneumonia. J Immunol 159(6): 28582866, 1997. PMID: 9300709.

Received March 6, 2020

Revised April 30, 2020

Accepted May 4, 2020 